\title{
From neutral to salt cocrystal development to gain superior performance of NSAIDs
}

\author{
I. Nugrahani ${ }^{1}$, H. Uekusa ${ }^{2}$, A. Horikawa², Felicia Fisandra ${ }^{1}$, R. A. Kumalasari ${ }^{1}$

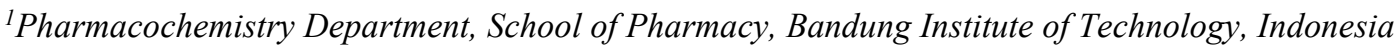 \\ ${ }^{2}$ Department of Chemistry, School of Sciences, Tokyo Institute of Technology, Japan \\ ilma_nugrahani@fa.itb.ac.id
}

Salt and cocrystal have been reported as two main classes of the solid phase to modulate the physicochemical properties of the active pharmaceutical ingredient (API), such as increased solubility, dissolution rate, and stability. Structurally, cocrystals are composed of neutral compounds; meanwhile, salt combines the ionic components. Each solid form offers advantages and can be tailormade to gain a specific purpose. For example, cocrystals may enhance solubility and dissolution rate, but some may also decrease those parameters. On the other hand, alkaline-drug salt generally has a higher solubility than the parent drug. But how if the alkaline salt drug is combined with a similar coformer? Can it produce a salt cocrystal with superior performance?

This poster presents the development of the two most used NSAIDs (non-steroidal anti-inflammatory drugs): diclofenac acid and mefenamic acid, with a scheme shown in Fig. 1. Previously, we have found and developed neutral cocrystals of diclofenac-L-proline [1] and mefenamic - nicotinamide [2]. However, the solubility was still lower than their alkaline salt forms. Hereafter, we attempted to combine the alkaline salt with a similar coformer to modulate its performance. As a result, we successfully produced the new salt cocrystals of diclofenac and mefenamic, characterized using DSC/TG, PXRD, and structurally determined using SCXRD entirely.

First, sodium and potassium diclofenac proline (NDP/KDP) were isomorphous and had two hydrate forms, monohydrate and tetrahydrate phases [3,4]. Meanwhile, sodium mefenamate nicotinamide (SMN) constructed the hemihydrate and monohydrate forms

[5]. The water's existence was crucial to stabilizing the lattice structure, coordinated with the alkaline elements and hydrogen-bonded with the other component. After that, all salt cocrystals were proven to modulate the physicochemical properties of the parent drugs, superior to the neutral cocrystals. The solubility and dissolution rate were increased by combining $\mathrm{Na}^{+}$and the soluble coformer. Furthermore, the lower hydrate forms showed a higher solubility, dissolution rate, and stability than the counterpart phases. NDP monohydrate and SMN hemihydrate increased the solubility of the starting alkaline salts by 3.5 and 1.5 folds or ten to a hundred times the acid parent drug's solubility, respectively. Finally, the powder properties of both new multi-components also were better than the parent and salt drugs. In conclusion, salt cocrystallization is a promising technique to improve the NSAIDs performance and can be developed further for the dosage form formulation.

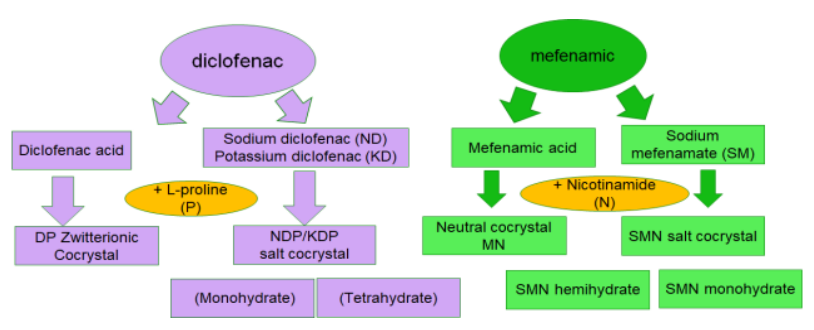

Figure 1: Salt cocrystal development of diclofenac acid and mefenamic acid.

[1] Nugrahani, I., Utami, D., Ibrahim, S., Nugraha, Y.P., Uekusa, H. (2018). Eur. J. Pharm. Sci. 117, 168.

[2] Utami, D.W.I., Nugrahani, I., Ibrahim, S. (2017). Asian J. Pharm. Clin. Res. 10, 135.

[3] Nugrahani, I., Kumalasari, R.A., Auli, W.N., Horikawa, A., Uekusa, H. (2020). Pharmaceutics 12, 690.

[4] Nugrahani, I., Komara, S.W., Horikawa, A., Uekusa, H. (2020). J. Pharm. Sci.109, 3423.

[5] Nugrahani, I., Fisandra, F., Horikawa, A., Uekusa, H. (2021). J. Pharm. Sci. available online 6 June 2021.

Keywords: salt cocrystal, solubility, dissolution, sodium/potassium diclofenac proline, sodium mefenamate nicotinamide.

We gratefully thank the Research and Innovation Institution, Educational Ministry of Republic Indonesia for the funding and Uekusa's Laboratory Tokyo Institute of Technology for the research collaboration. 